\title{
Gradhiva
}

GRADHIV

Revue d'anthropologie et d'histoire des arts

\section{À la marge des sciences coloniales ? La mission Dekeyser-Holas dans l'Est libérien (1948)}

At the limits of the colonial sciences? The Dekyser-Holas expedition in Eastern Liberia (1948)

Julien Bondaz

\section{OpenEdition}

\section{Journals}

Édition électronique

URL : http://journals.openedition.org/gradhiva/3074

DOI : 10.4000/gradhiva.3074

ISSN : 1760-849X

\section{Éditeur}

Musée du quai Branly Jacques Chirac

Édition imprimée

Date de publication : 1 octobre 2015

Pagination : 168-191

ISBN : 978-2-35744-092-0

ISSN : 0764-8928

\section{Référence électronique}

Julien Bondaz, «À la marge des sciences coloniales ? La mission Dekeyser-Holas dans l'Est libérien (1948) », Gradhiva [En ligne], 22 | 2015, mis en ligne le 01 octobre 2018, consulté le 20 avril 2019. URL : http://journals.openedition.org/gradhiva/3074; DOI : 10.4000/gradhiva.3074 


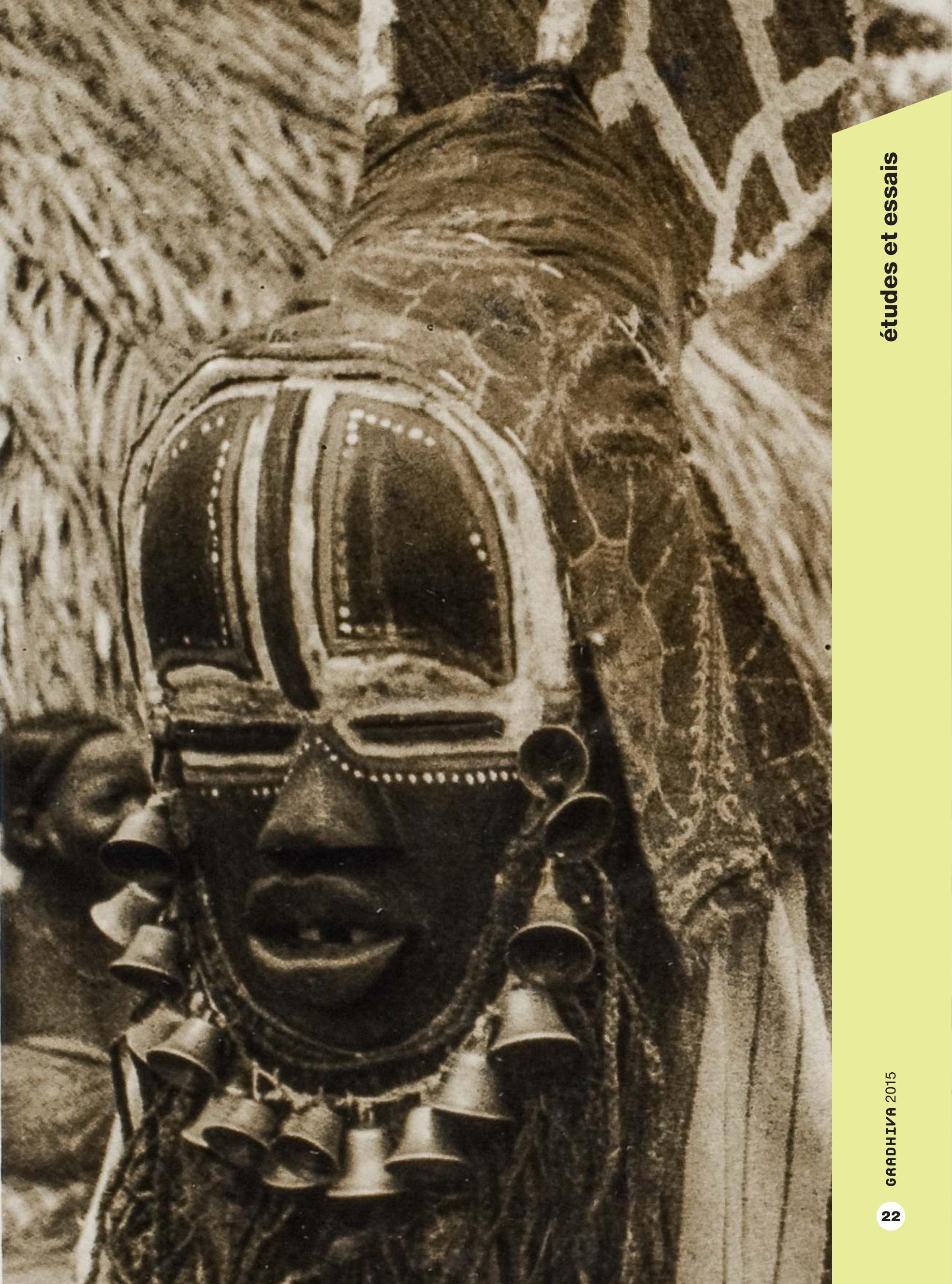




\section{À la marge des sciences coloniales?}

La mission Dekeyser-Holas dans l'Est libérien (1948)

par Julien Bondaz

En 1948, une mission de recherche et de collecte est organisée par l'Institut français d'Afrique noire (IFAN) à l'initiative de Théodore Monod. Conduite par un ethnologue et un zoologiste, Bohumil Holas et Pierre Louis Dekeyser, elle fournit un cas tout à fait intéressant de mission bidisciplinaire, permettant d'interroger la mixité des pratiques de terrain et les interférences entre sciences sociales et sciences naturelles qui en résultent. La mission Dekeyser-Holas dans l'Est libérien est aussi l'une des rares missions coloniales qui se déroulent dans un pays indépendant. Interroger ce paradoxe oblige ainsi à resituer ces recherches et ces collectes dans le contexte institutionnel et scientifique de l'IFAN à la sortie de la Seconde Guerre mondiale. Décrire et analyser cette mission permet alors d'éclairer de manière inédite les enjeux politiques et disciplinaires de l'ethnographie et de la zoologie à la fin de la période coloniale. 


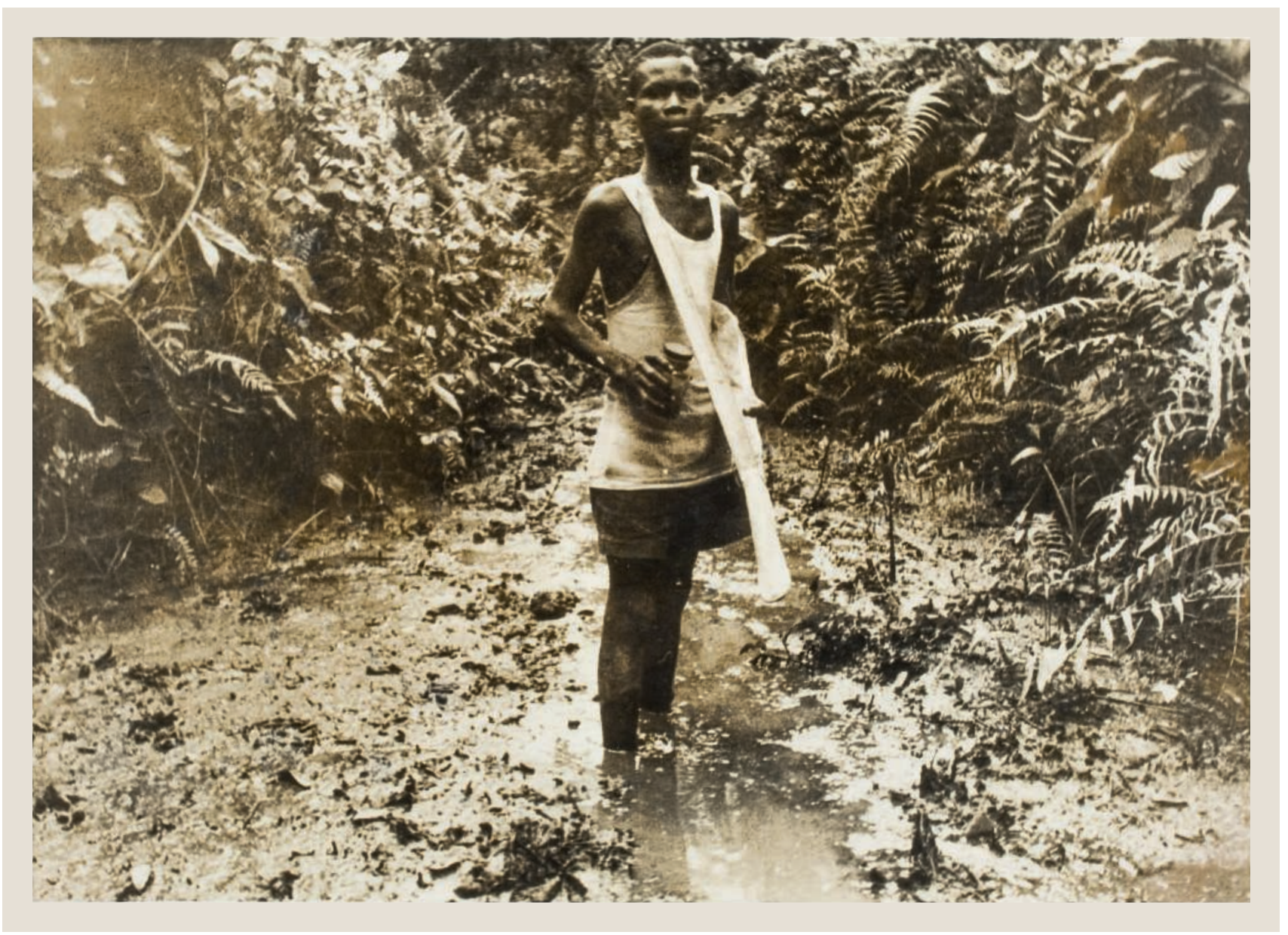

fig. 1

Récolte d'insectes. Mission IFAN DekeyserHolas au Libéria en 1948. Photographie Bohumil Holas (C) musée du quai Branly.
Au sortir de la Seconde Guerre mondiale, I'Institut français d'Afrique noire (IFAN), fondé en 1936 et basé à Dakar, prend un certain essor sous l'impulsion de son directeur, Théodore Monod ${ }^{\mathbf{1}}$ (Adedze 2003). Plusieurs sections de recherche sont créées ou dynamisées. Celle de zoologie par exemple, mise en place en 1943, devient véritablement fonctionnelle trois ans plus tard avec l'arrivée à sa tête de Pierre Louis Dekeyser. Les effectifs de l'institut s'accroissent, de même que le nombre de boursiers de l'École française d'Afrique. Bohumil Holas, ethnologue tchèque formé à l'université de Prague, en devient membre en juin 1946, pour une durée de deux ans ${ }^{2}$. De grands chantiers sont initiés, notamment une importante enquête «ethnogéographique» (Suremain 2005 et 2008), ainsi que plusieurs tentatives visant à fédérer les chercheurs africanistes au-delà du cadre territorial des colonies françaises. C'est en particulier le cas de la première Conférence internationale des africanistes de l'Ouest, organisée à Dakar en 1945 (Gouvernement général de l'AOF-IFAN 1950 et 1951). Enfin, de nombreux centres locaux de I'IFAN, ou Centrifans, sont créés dans les capitales des différentes colonies de l'Afrique occidentale française afin «d'assurer une sorte de quadrillage scientifique du territoire» (L'Estoile 2007: 125). En 1948, Monod esquisse ainsi «la silhouette d'un IFAN nouveau, étendu à l'ensemble de l'Afrique noire française et au sein duquel les organismes régionaux de recherche conserveront leur autonomie administrative, tout en participant à un effort 
commun enfin libéré de l'esclavage des frontières et des misères de l'esprit de clocher» (Monod 1948a: 29). Les centres locaux sont alors considérés comme des «avant-postes de brousse» (Monod 1943: 198), la métaphore militaire témoignant de la volonté de faire avancer les frontières de la zone d'influence de l'IFAN et de ses terrains de recherche.

Dans un tel contexte, l'organisation de missions scientifiques de plus en plus nombreuses, conduites par le personnel de I'IFAN ou par les membres de l'École française d'Afrique, rend bien compte du développement de l'activité de l'institut sous l'impulsion de Monod ${ }^{3}$. Parmi les nombreuses missions scientifiques organisées au sortir de la Seconde Guerre mondiale, celle conduite au Libéria en 1948 par le zoologiste Dekeyser et l'ethnologue Holas est intéressante à plus d'un titre ${ }^{4}$. C'est d'abord l'une des rares missions organisées hors du territoire colonial français, qui plus est dans la première nation africaine à avoir obtenu son indépendance, en 1847. On peut dès lors se demander dans quelle mesure cette recherche menée hors situation coloniale peut nous éclairer sur la politique de l'IFAN et sur la «localisation» des sciences coloniales (Chambers et Gillespie 2000; Seth 2009). Si l'on considère que ces dernières englobent à la fois les savoirs scientifiques produits par des Européens dans les territoires coloniaux et ceux construits en Europe sur la base de collections et de données récoltées dans les colonies (Schiebinger 2005: 52), comment situer les connaissances produites par les deux chercheurs de l'IFAN dans l'Est libérien?

La mission Dekeyser-Holas est en outre l'une des rares missions mises en place par l'institut qui rassemble un chercheur en sciences naturelles et un chercheur en sciences sociales, en l'occurrence un zoologiste et un ethnologue. En ce sens, elle constitue un cas privilégié pour l'étude des affinités ou des interférences entre zoologie et ethnologie dans les manières de faire du terrain en Afrique à la fin de la période coloniale. On peut en effet se demander dans quelle mesure cette conjonction des approches et des pratiques a des conséquences sur les plans méthodologique, théorique et épistémologique. Cette mission fournit aussi l'occasion d'interroger la polyvalence des chercheurs de I'IFAN, voire la pluridisciplinarité de l'institut, telles qu'elles ne cessent d'être mises en avant dans les textes programmatiques. Enfin, cette mission itinérante, effectuée le plus souvent à pied sur un total de près de 900 kilomètres de pistes pendant six mois, permet à Holas de mener des enquêtes dans 165 villages. Celui-ci précise d'ailleurs qu'«il ne s'agit que d'une prospection ${ }^{5}$ ». La logique est celle de l'inventaire et de l'enquête extensive, dans la continuité des tournées qu'il a effectuées en 1946-1947 pour le compte de l'IFAN, en «complément au Répertoire des populations de l'AOF ${ }^{6}$ ». La mission s'inscrit dans le cadre du programme d'«ethnologie générale et religieuse» ou d'«ethnologie comparée» de Holas, et les données qu'il rassemble au Libéria sont donc nombreuses et variées, mais largement superficielles. Cette ethnographie «au sens large» témoigne ainsi des enjeux et des limites du programme scientifique et des ambitions de l'IFAN au sortir de la Seconde Guerre mondiale, et plus largement du dialogue entre les disciplines et de l'influence du modèle naturaliste sur les méthodes de l'ethnographie.
1. Les données présentées dans cet article sont issues de recherches menées dans les archives de l'Institut fondamental d'Afrique noireCheikh Anta Diop à Dakar et dans celles du musée du quai Branly à Paris. Je tiens à remercier Hamady Bocoum, Thiéyacine Ngom et Sarah Frioux-Salgas pour leur assistance lors de ces recherches.

2. L'École française d'Afrique est une section de I'IFAN qui accueille des boursiers nommés pour deux ans par le gouverneur général de l'Afrique occidentale française. Comme le précise l'arrêté nº 1945 créant l'IFAN, «peuvent [...] être désignés soit des jeunes gens se destinant à l'étude de l'Afrique noire qui pourraient offrir des garanties sérieuses de préparation scientifique, soit des savants dont les recherches rendent indispensable un séjour en Afrique noire ". Le cas de Bohumil Holas relève clairement de la première catégorie.

3. Pour ce qui concerne les «missions des membres de l'École française d'Afrique ", la première est organisée en 1942, et dix-sept sont menées entre 1945 et 1948.
4. Sans compter que les nombreux travaux concernant les liens entre science et colonialisme sont majoritairement consacrés à la période qui va du XIX ${ }^{e}$ siècle aux années 1930, I'histoire des acteurs réseaux, institutions et missions scientifiques de la fin de la période coloniale n'ayant retenu l'attention qu'exceptionnellement, et plus récemment. Sur ce point, voir Jézéquel 2011.

5. Lettre de Bohumil Holas à Théodore Monod, 29 janvier 1948, dossier E2.7, archives de l'IFAN-CAD.

6. Ordre de service du 23 septembre 1946 , dossier E2.7, archives de I'IFAN-CAD. 
7. Le National Museum of Liberia est finalement créé en 1958 à Monrovia, et ses collections ne se développent qu'à partir des années 1960.

8. Sur les premières missions aux monts Nimba, voir Lamotte, Roy et Xavier 2003.

\section{ci-contre}

fig. 2

Itinéraire de la mission P.L.

Dekeyser et B. Holas de

1948, in Holas 1952, p.14.

\section{Une recherche périphérique}

La mission Dekeyser-Holas est organisée sous l'impulsion directe de Monod, qui a commencé à nouer des liens étroits avec le gouvernement du Libéria à partir de 1947. En cette année du centenaire de l'indépendance du pays, le directeur de l'IFAN se rend une première fois à Monrovia pour participer aux cérémonies, puis une seconde pour travailler à un projet de création de musée national avec le gouvernement libérien ${ }^{7}$. Sur le plan de la recherche, il rédige à cette occasion un bilan des études sur le Libéria (Monod 1947) et initie un vaste projet de bibliographie générale en lançant un appel dans la revue Man (Monod 1948b). L'idée d'organiser une mission de recherche au Libéria est alors avancée, et Monod obtient, en novembre 1947, un «accord de principe» du gouvernement libérien. Pour lui, l'organisation de cette mission vise ainsi un double objectif: il s'agit à la fois de compléter les connaissances concernant les régions voisines de la Côte d'Ivoire et de la Haute-Guinée et de mettre en place un partenariat privilégié avec le Libéria.

La mission concerne I'hinterland libérien, c'est-à-dire la partie orientale du pays. Le choix du terrain, ou plus exactement de l'itinéraire de recherche, s'explique par plusieurs motifs. La zone est frontalière avec la Guinée française et la Côte d'Ivoire, et constitue ainsi ce que Holas appelle «les confins AOF Libéria». Cela revient à prendre acte de l'artificialité des frontières ethniques, à la fois sur le plan ethnodémographique et sur le plan biogéographique. Sur le premier plan, l'un des objectifs centraux de la mission est de «compléter la carte ethnique et le répertoire des populations de l'Afrique occidentale». Durant les préparatifs de la mission au centre IFAN d'Abidjan, Holas est d'ailleurs en correspondance étroite avec Jacques RichardMolard, le géographe chargé de l'enquête ethnodémographique à la base de Dakar. L'idée centrale est alors de comparer les «races» de part et d'autre du Cavally, le fleuve qui fait office de frontière entre le Libéria et la Côte d'Ivoire, ce qui explique l'importance que vont prendre, dans le travail de terrain de l'ethnologue et dans la publication de ses résultats, les données relatives à la géographie humaine, à l'anthropométrie et à la linguistique.

De la même manière, l'idée selon laquelle les frontières politiques «n'ont évidemment aucune réalité biogéographique» (Dekeyser 1955: 14) est mise en avant par Dekeyser, qui entend compléter l'inventaire de la faune de l'Afrique occidentale. Cette idée doit bien sûr être mise en relation avec la création, en 1944, de la réserve intégrale des monts Nimba, qui se situe précisément à l'intersection des frontières de la Guinée française, de la Côte d'Ivoire et du Libéria et qui constitue «un véritable carrefour climatique et écologique» (IFAN 1954: 938). Dans les programmes ethnologiques et zoologiques, on retrouve ainsi un même argument reposant sur l'artificialité des frontières politiques et une même logique d'inventaire. De façon évidemment problématique, deux «populations», l'une humaine et l'autre faunistique, font l'objet d'une même logique d'inventaire.

La nécessité d'inventorier les groupes humains et la faune de l'Est libérien explique le choix d'une enquête extensive plutôt qu'intensive, choix renforcé par l'absence ou la faiblesse des connaissances publiées jusque-là sur cette zone. La seule mission scientifique d'importance organisée aupa- 


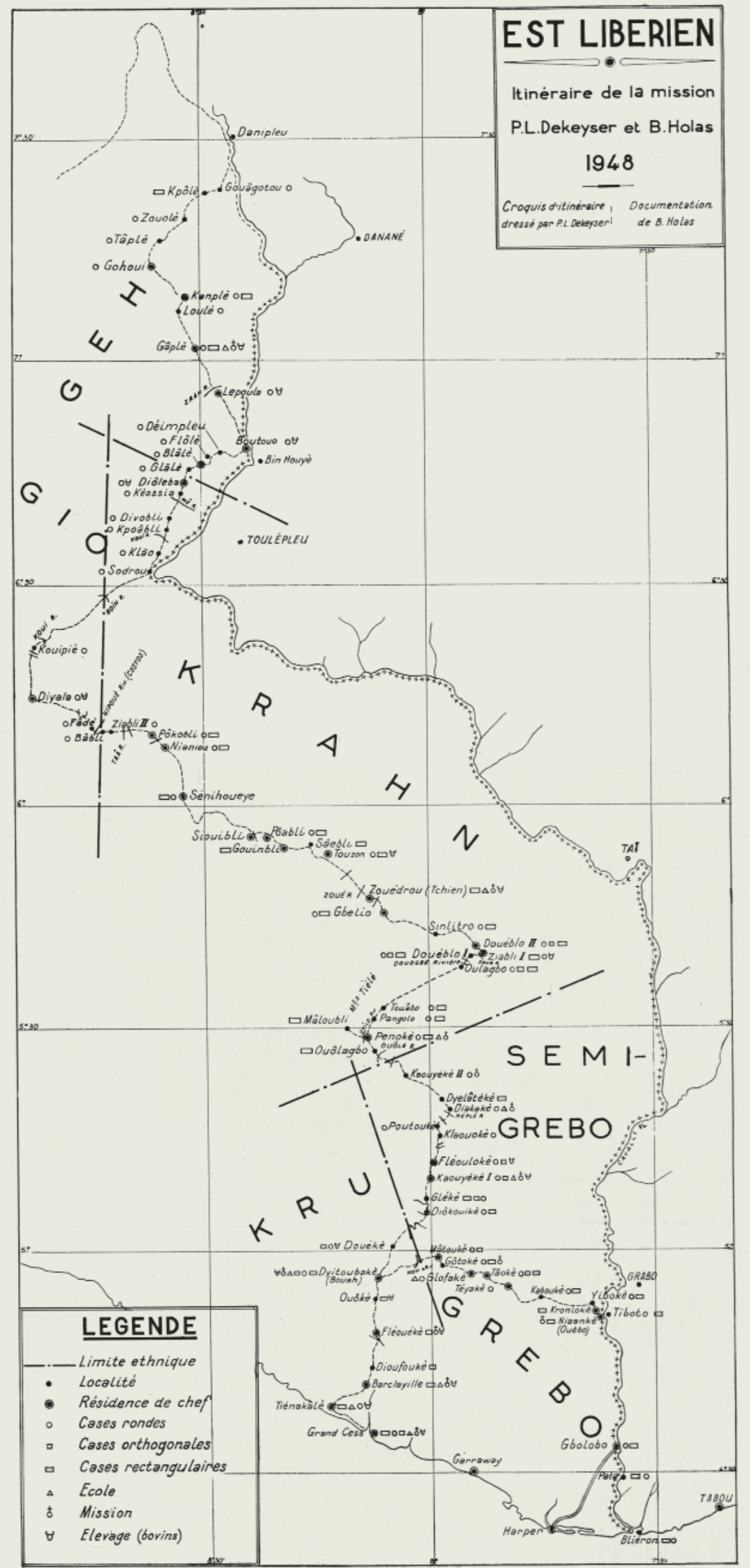


9. Sir Harry Johnston, naturaliste en poste dans l'administration coloniale, fait cependant paraître deux volumes de synthèse sur le Libéria en 1906 (Johnston 1906) et, en 1926, un an après avoir créé d'importantes plantations de caoutchouc dans le pays, l'industriel américain Firestone finance, avec la fondation Rockefeller et l'université Harvard, une expédition conduite par Richard P. Strong, de la Harvard Medical School (Strong [éd.] 1930). Sur les liens entre l'avancée de la science et l'industrie américaine au Libéria, voir McBride 2002 : chap. IV.

10. Rapport de mission du 29 juin 1948, dossier E2.7, archives de I'IFANCAD : 3-4. Voir aussi Mahé 1958: 21.

11. Holas note: "Mais au village de Mãdwa nous avons rencontré quelques vieux portant des traces négroïdes" (sur ces «négrilles », voir Holas 1952: 113-115; Schnell 1948).

12. Rapport intérieur, dossier E2.7, archives de l'IFAN-CAD : 4

13. Ibid. : 3-4 ravant dans l'Est libérien était celle conduite par le zoologiste suisse Johann Büttikofer entre 1879 et 1882, puis en 1886-1887, pour le compte du Muséum royal d'histoire naturelle de Leyde ${ }^{9}$ (Büttikofer 1890). Holas met donc en avant le fait qu'il s'agit d'«un territoire jusqu'à ce jour scientifiquement non exploité ${ }^{\mathbf{1 0}}$ ». L'idée d'une tache blanche sur la carte est plusieurs fois avancée, la mission Dekeyser-Holas visant d'ailleurs également à compléter la cartographie. La méthode itinérante ou extensive revendiquée par les chercheurs trouve ainsi pour arrière-plan les récits d'exploration, dont témoignent, dans les rapports rédigés par Holas, la mise en avant des difficultés liées au terrain et l'insistance sur les bourbiers à traverser ou les pistes à ouvrir. L'ethnologue envisage même de se plaindre auprès de I'IFAN pour la dotation insuffisante en chaussures... Les affinités entre mission scientifique et exploration culminent - si l'on peut dire - avec l'ascension, le 3 avril 1948, du mont Tiélé, considérée comme sa première ascension. Dekeyser et Holas proposent d'ailleurs d'appeler cette montagne de 575 mètres d'altitude mont ou pic Théodore Monod, en hommage à l'initiateur de la mission (Holas 1952: 110113 , croquis p. 111). L'enquête que les deux chercheurs mènent concernant l'éventuelle présence (ou les souvenirs de cette présence) de gorilles pour le premier, de Pygmées pour le second, dans cette montagne considérée comme sacrée témoigne bien des confusions possibles entre enjeux scientifiques et récits d'exploration ${ }^{\mathbf{1 1}}$.

Au-delà des spécificités du terrain, les difficultés rencontrées par la mission sont également liées à la situation politique du Libéria et notamment aux relations difficiles avec les responsables administratifs locaux, contre lesquels Holas multiplie les plaintes dans ses rapports internes. Malgré l'autorisation accordée par le gouvernement libérien, les papiers de la mission ne sont d'ailleurs pas en règle. L'ethnologue résume en ces termes les difficultés administratives rencontrées à la frontière du Libéria:

En réalité, aucun Blanc n'est admis dans l'intérieur, exclusion faite de quelques missionnaires, jugés sans doute inoffensifs et acceptés au même titre que les plantations de caoutchouc. Si les autorités supérieures ont consenti à faire une exception [...], les autorités secondaires ne tenaient sans doute pas à ce que des étrangers, et, par surcroît des Blancs, se rendent compte de l'organisation et de l'état des territoires qu'elles administrent ${ }^{12}$.

Holas indique alors que les deux chercheurs ont été contraints d'entrer au Libéria «par effraction». II se plaint en outre de l'«amour immodéré du "dash" ", ou "cadeaux", que manifestent les administrateurs locaux ou les chefs de village, et des difficultés rencontrées lors du recrutement des porteurs (le faible nombre de porteurs contraint le plus souvent l'ethnologue et le zoologiste à suivre un itinéraire commun, contrairement à ce qui était prévu). II insiste également sur la méfiance des populations locales à l'égard de la mission: "enquête effectuée dans une atmosphère de méfiance aiguë", «accueil peu hospitalier», "l'hostilité, la mauvaise volonté, la cupidité et le mensonge ont constitué l'ambiance dans laquelle se déroulaient nos enquêtes" (Holas 1952). Enfin, il accuse le guide mis à la disposition de la mission par le superintendant du Maryland Country, non seulement de ne pas respecter les ordres, mais surtout d'«être un agent de contrôle ${ }^{13}$ ». On voit 
bien ici comment les conditions de recherche hors situation coloniale tendent à renverser les rapports de pouvoir entre chercheurs «blancs » et population «noire» (Holas insiste à plusieurs reprises sur cette distinction). Dès lors, pour l'ethnologue, la recherche en territoire libérien ne doit plus seulement permettre de «gagner de nouvelles connaissances»: il s'agit également de «faire pénétrer un peu de civilisation française dans ce pays corrompu ».

La recherche menée en pays étranger pose également la question des interférences entre la figure de l'enquêteur et celle de l'espion. RichardMolard, dans sa correspondance avec Holas, lui recommande ainsi une certaine discrétion, indiquant: «estimation par vous du nombre de cases des villages où vous passez et comparaison avec le chiffre officiel fourni par la hut tax (en gardant bien pour vous ces comparaisons indiscrètes ${ }^{\mathbf{1 4}}$ !) "). Et l'ethnologue note pour sa part qu'«étant donné la véracité douteuse des renseignements fournis au cours des enquêtes officielles [...], nous avons procédé ultérieurement à une enquête privée de contrôle" (Ibid.: 138 , note 1 ) et qu'il privilégie le recours aux «informateurs non officiels». De ce point de vue, l'idée selon laquelle la publication des résultats de l'enquête menée par Holas «rendra service [...] à l'administration libérienne elle-même», idée avancée par Monod dans sa préface (Monod, in ibid. : VIII), apparaît paradoxale. On retrouve d'une part l'articulation entre la production des savoirs et la rationalisation de l'administration. On devine d'autre part que les relations conflictuelles avec les autorités libériennes locales brouillent les perspectives d'avenir de la recherche française en territoire libérien.

\section{Ethnologie et zoologie}

Des missions de recherche collectives et pluridisciplinaires sont souvent organisées par l'IFAN, mais la frontière entre sciences naturelles et sciences humaines et sociales reste très largement respectée. Holas a par exemple participé à une mission de recherche avec Georges Balandier et Paul Mercier sur les pêcheurs lébou dans la région de Dakar (Balandier et Mercier 1952). Dekeyser a pour sa part l'habitude de partir sur le terrain avec André Villiers, l'entomologiste de l'institut. Ce dernier, à propos de l'une de ces missions communes, parle ainsi d'«une équipe fraternelle dans laquelle Européens et Africains œuvrent ensemble d'un même cœur et où chacun est avant tout, plutôt qu'un "spécialiste", un "naturaliste", de sorte que, suivant les circonstances, le taxidermiste traque les papillons, le mammalogiste manie l'aspirateur et les entomologistes le fusil » (Villiers 1949: 8). L'idée selon laquelle une telle polyvalence des chercheurs pourrait être plus large encore est pourtant fréquemment avancée. Le géographe Jean Dresch note par exemple: «Nos connaissances sont trop incomplètes pour que les efforts ne soient pas rassemblés, et qu'à l'exemple de Th[éodore] Monod un naturaliste ne dédaigne pas de noter gravures rupestres, légendes et outils, un ethnologue ne renonce pas à ramasser plantes, insectes ou cailloux. » (Dresch 1951 : 225) Mais ces bonnes volontés et ces appels ne débouchent pas sur une véritable pluridisciplinarité (Suremain 2004 : 653).

L'organisation d'une mission rassemblant un zoologiste et un ethnologue se révèle donc de ce point de vue particulièrement intéressante à analyser. Certes, Holas parle d'une équipe «séparée en deux camps par
14. Lettre de Jacques Richard-Molard à Bohumil Holas, 2 octobre 1947 dossier E2.7, archives de I'IFAN-CAD. 
15. Pierre Louis Dekeyser et Bohumil Holas, "Rapport de mission $n^{\circ} 1$ ", 19 février 1948, dossier E2.7, archives de I'IFAN-CAD.

16. Carnet "Mission Libéria-1948 ", 17 mars 1948, archives du musée du quai Branly.

17. Rapport de mission du 29 juin 1948, op. cit. : 3. Sur les collectes entomologiques menées à la même époque par des ethnologues africanistes, voir Bondaz 2013. la nature même des recherches " (Holas 1952: X), mais sur le terrain, notamment à cause des contraintes de portage évoquées plus haut, les deux chercheurs travaillent souvent ensemble, «dans un climat de collaboration réciproque ${ }^{15}$ ». On peut ainsi se demander dans quelle mesure zoologie et ethnologie ne se rencontrent pas autour d'un même «paradigme cynégétique» (Bondaz 2011 et 2015). Un même objet, les poteaux à trophées par exemple, permet à Dekeyser toute une série d'observations et de déterminations scientifiques et lui fournit des indices concernant le peuplement animal, notamment la répartition de l'hippopotame nain. II note ainsi : «Dans de nombreux villages nous avons trouvé des fragments de crânes, parfaitement identifiables, en maintes occasions. » (Dekeyser 1955: 333) Holas analyse pour sa part ces poteaux à trophées comme des témoignages de la vie religieuse des villageois. À de nombreuses reprises, le zoologiste et l'ethnologue collaborent dans ce travail de détermination des crânes exposés sur les poteaux à trophées, en particulier pour ce qui concerne les animaux totémiques (Holas 1952: 151).

En outre, l'intérêt accordé par Holas aux différents cultes totémiques ou à la chasse rituelle aux potamochères chez les Plapo trouve sans doute une part d'explication dans le goût qu'il a lui-même pour la chasse. Ses carnets de terrain en Côte d'Ivoire sont remplis d'informations concernant les zones giboyeuses ou les aménagements susceptibles de favoriser le tourisme cynégétique. Un tel goût pour la chasse explique également que l'ethnologue profite des «temps perdus » au cours de la mission pour augmenter les collections zoologiques, se plaignant d'ailleurs à ce sujet d'être doté d'un «mauvais fusil (qui est indispensable ${ }^{16}$ !) ». II récolte en outre un grand nombre d'insectes ${ }^{\mathbf{1 7}}$. Dekeyser et lui rapportent ainsi à I'IFAN 250 mammifères et oiseaux, 12 bocaux de vertébrés inférieurs et d'invertébrés et 8500 insectes, dont 3500 papillons. De nombreux objets ethnographiques sont également rassemblés, tandis qu'un projet d'herbier est abandonné. Les spécimens de petite taille sont privilégiés à cause des contraintes de portage. La pratique de ces collectes mixtes rend compte à la fois de la visée encyclopédiste de l'IFAN, qui reproduit en Afrique le modèle du Muséum national d'histoire naturelle de Paris, et du caractère extensif de la mission. Le cas des collections entomologiques témoigne cependant de certaines limites: ni Holas ni Dekeyser ne sont spécialistes de cette discipline, ce qui explique qu'à leur retour l'entomologiste de l'IFAN regrette que les petits insectes aient été négligés au moment de la collecte (Villiers 1950: 930). Quoi qu'il en soit, la chasse et les récoltes zoologiques ou entomologiques constituent pour les deux chercheurs une activité partagée tout au long de la mission.

Le récit que Dekeyser fait de l'une de leurs acquisitions (manquées) est de ce point de vue révélateur des pratiques des deux chercheurs sur le terrain:

Au cours de notre mission sur les confins éburnéo-libériens (1948) nous avons eu l'occasion de collecter à Grabo (Côte d'Ivoire), à la suite d'un heureux coup de fusil de notre ami B[ohumil] Holas, un grand anomalure dont le pelage était uniformément noir, sans aucune marque blanche. La caisse qui le contenait fut malheureusement perdue au cours de son transport par mer sur Dakar. Nous n'avons 


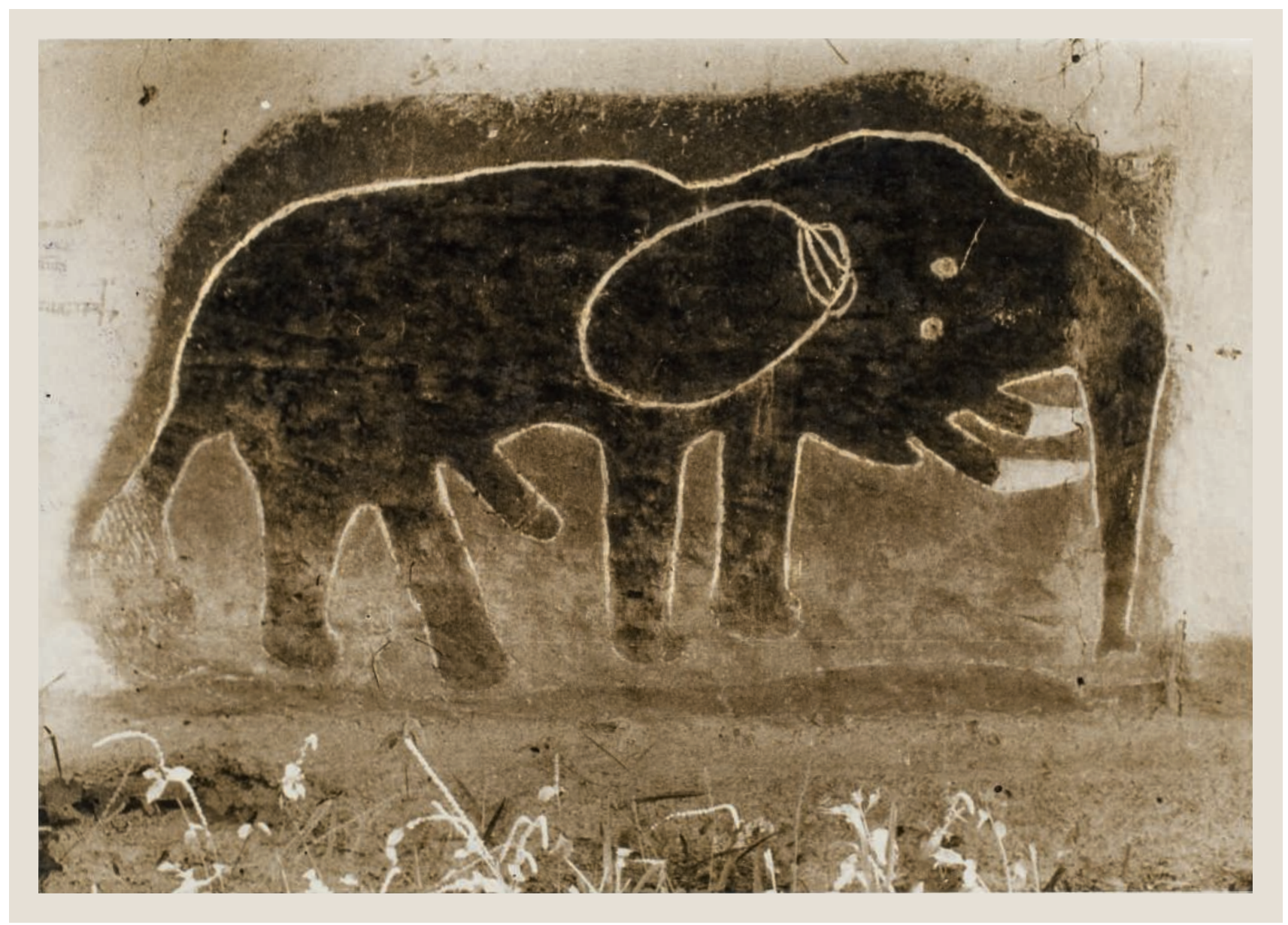

fig. 3

Un éléphant, dessin mural.

Mission IFAN Dekeyser-

Holas au Libéria en 1948.

Photographie Bohumil

Holas (C) musée du quai

Branly. 
178

études et essais

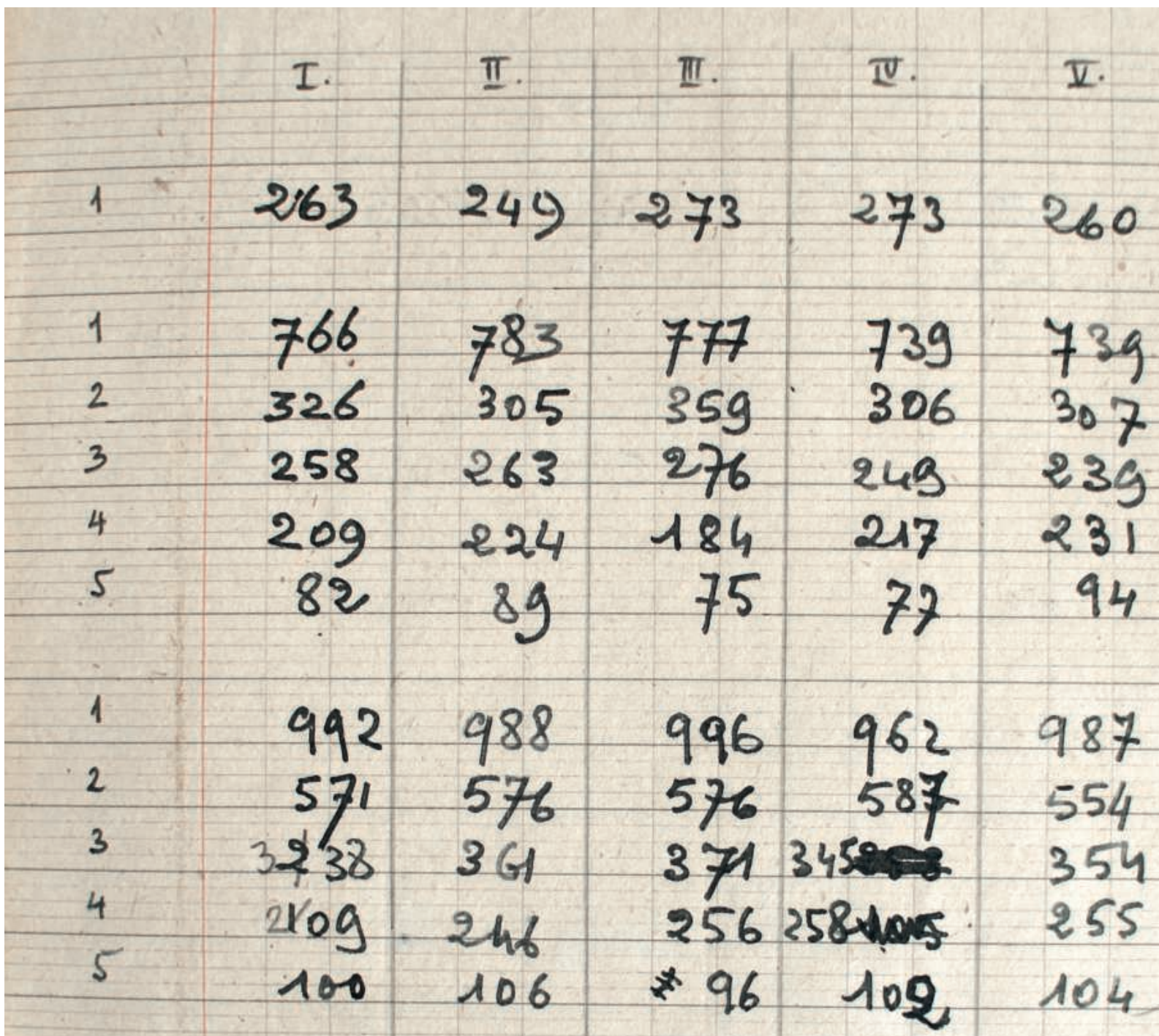

Note:

1) $\theta$

1) $\theta$

1)

1) $\theta$

1) $\theta$

2) $\rightarrow$

2)

2) $\rightarrow$

(iii

2)

3) 2

$\frac{\pi}{201}$

2) $\rightarrow$

3)

4) $\rightarrow$

3) $\theta$

3) -

4)

4) tat.

4) $a$.

sulles

2 Av. Gias 
plus de cette pièce que la photographie que nous publions. Les Kru donnent à l'animal le nom de poê.

$[\ldots]$

Il n'est pas d'usage, et en tout cas nullement souhaitable, de donner un nom spécifique à un animal dont on ne possède ni peau ni crâne. Cependant, nous considérons que l'anomalure de Grabo représente plutôt une espèce inédite qu'un représentant anormal, mélanique, de l'anomalure de Pel. À ce propos, si quelque heureux coup de fusil permettait d'obtenir un nouveau spécimen, nous serions enchanté d'en recevoir la dépouille et la tête osseuse, aussi imparfaite qu'en soit la préparation. (Dekeyser 1954: 124)

Holas participe directement à la constitution des collections zoologiques, non seulement en abattant l'anomalure (une sorte d'écureuil), mais aussi en en photographiant la dépouille. L'importance accordée aux noms vernaculaires des animaux collectés témoigne en outre de l'intérêt que Dekeyser accorde aux savoirs zoologiques locaux, prémisse à la recherche ethnozoologique.

Le rôle joué par les auxiliaires africains dans la production des savoirs est ici essentiel. Non seulement Dekeyser et Holas sont accompagnés dans leur mission par un préparateur de l'IFAN, André Keita, mais les chasseurs locaux sont particulièrement sollicités. On retrouve ici l'importance que Dekeyser et Villiers conseillent d'accorder aux «auxiliaires indigènes» dans le guide qu'ils font paraître l'année même de la mission, intitulé Récolte et préparation des collections zoologiques (Dekeyser et Villiers 1948: 3). Les cuisiniers recrutés en Côte d'Ivoire par Holas sont interrogés au même titre que les indigènes du Libéria. Dekeyser note par exemple, à propos d'un crâne de céphalophe de Jentink, que sa distribution «ne paraît pas exclusivement libérienne. Nos cuisiniers ouobé (de la région de Man, Côte d'Ivoire) connaissaient ce céphalophe sous le nom de niénagbé, vocable très voisin du terme nyagbé employé par les gens de Klosoké. Le céphalophe de Jentink paraît bien faire partie d'un peuplement résiduel axé sur le Cavally, comme I'hippopotame nain et sans doute aussi le céphalophe zébré ${ }^{18}$. " (Dekeyser et Villiers 1955: 57) Une telle division du travail, où les auxiliaires des chercheurs, comme le personnel de la mission, sont à la fois des assistants et des informateurs, est d'ailleurs l'une des caractéristiques communes aux sciences naturelles et à l'ethnologie (L'Estoile 2005). L'occultation du rôle qu'ils jouent dans les activités de collecte ethnographique, mais aussi de capture ou de préparation des spécimens zoologiques (Jacobs 2006), rend bien compte des rapports asymétriques entre chercheurs et auxiliaires indigènes ${ }^{19}$.

\section{L'ethnologie lato sensu}

Les enquêtes ethnologiques menées par Holas lors de la mission reposent sur l'idée selon laquelle la méthode ethnographique doit être «appuyée par la géographie humaine, l'anthropologie physique, la linguistique» (Dieterlen 1952: 167). La publication des résultats de ses recherches rend bien compte de l'importance accordée à ces différentes approches. Le livre qu'il fait paraître en 1952 sous le titre Mission dans l'Est libérien (P.L. Dekeyser-B. Holas) est d'ailleurs sous-titré Résultats démographiques,
18. II est frappant de voir combien le croisement des données linguistiques et de celles qui concernent le peuplement faunistique, de même que l'hypothèse d'un peuplement résiduel, sont proches des analyses que Holas développe au sujet de la répartition des populations indigènes.

19. Les photographies prises lors de la mission sont révélatrices du statut accordé par les deux chercheurs aux indigènes qu'ils emploient.

Sur plusieurs figure

la longue colonne

de porteurs qui les

accompagne, donnant à voir la mission comme une véritable expédition, tandis que les rares portraits individuels restent anonymes, comme sur cette photographie où I'on voit un Libérien tenant dans ses bras une gazelle vivante, simplement titrée "Une gazelle".

\section{ci-contre}

fig. 4

Tableau de mensurations anthropométriques, cahier «Anthropométrie (Libéria 1948) - III ", fonds Bohumil Holas, musée du quai Branly. 


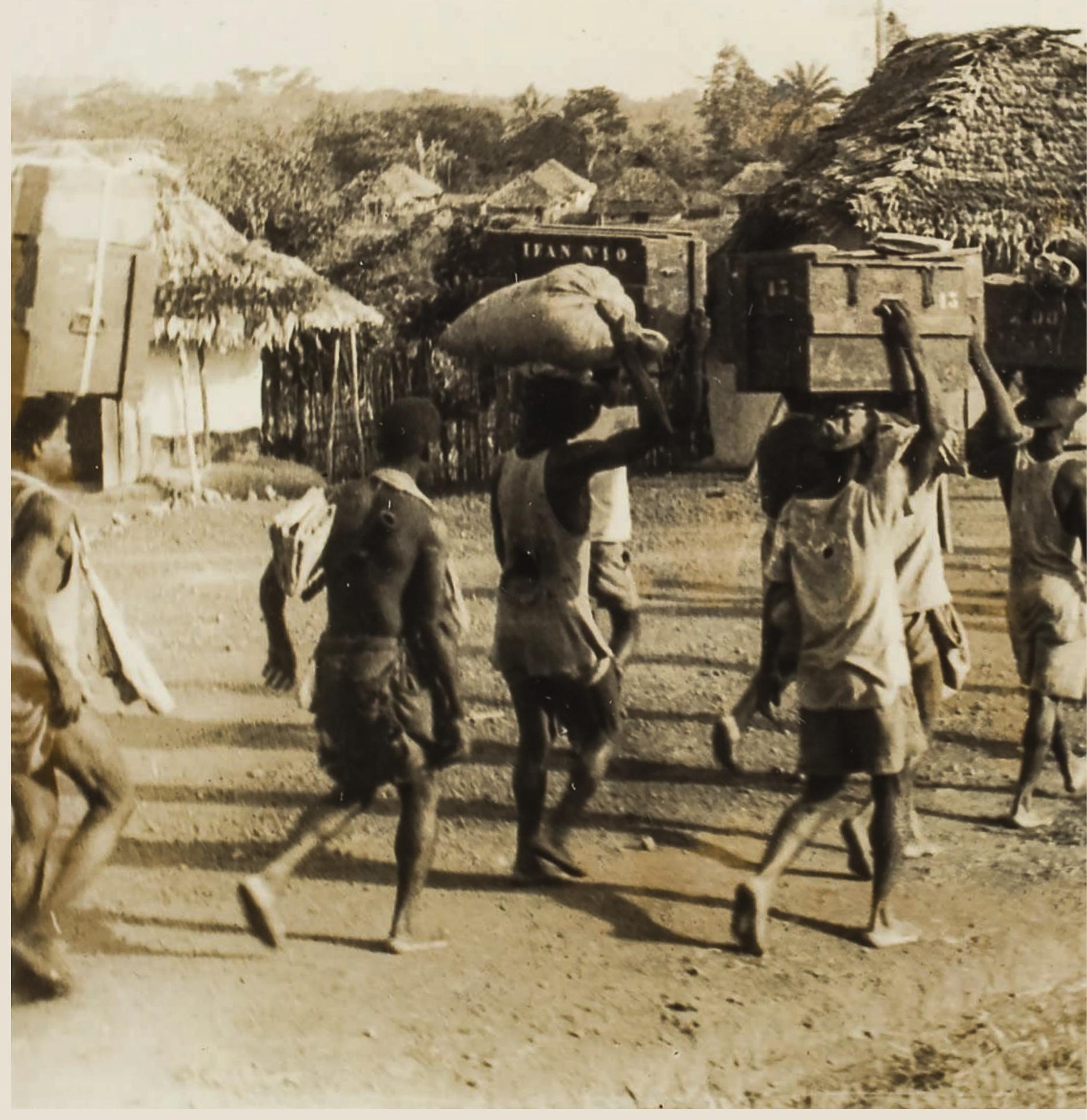




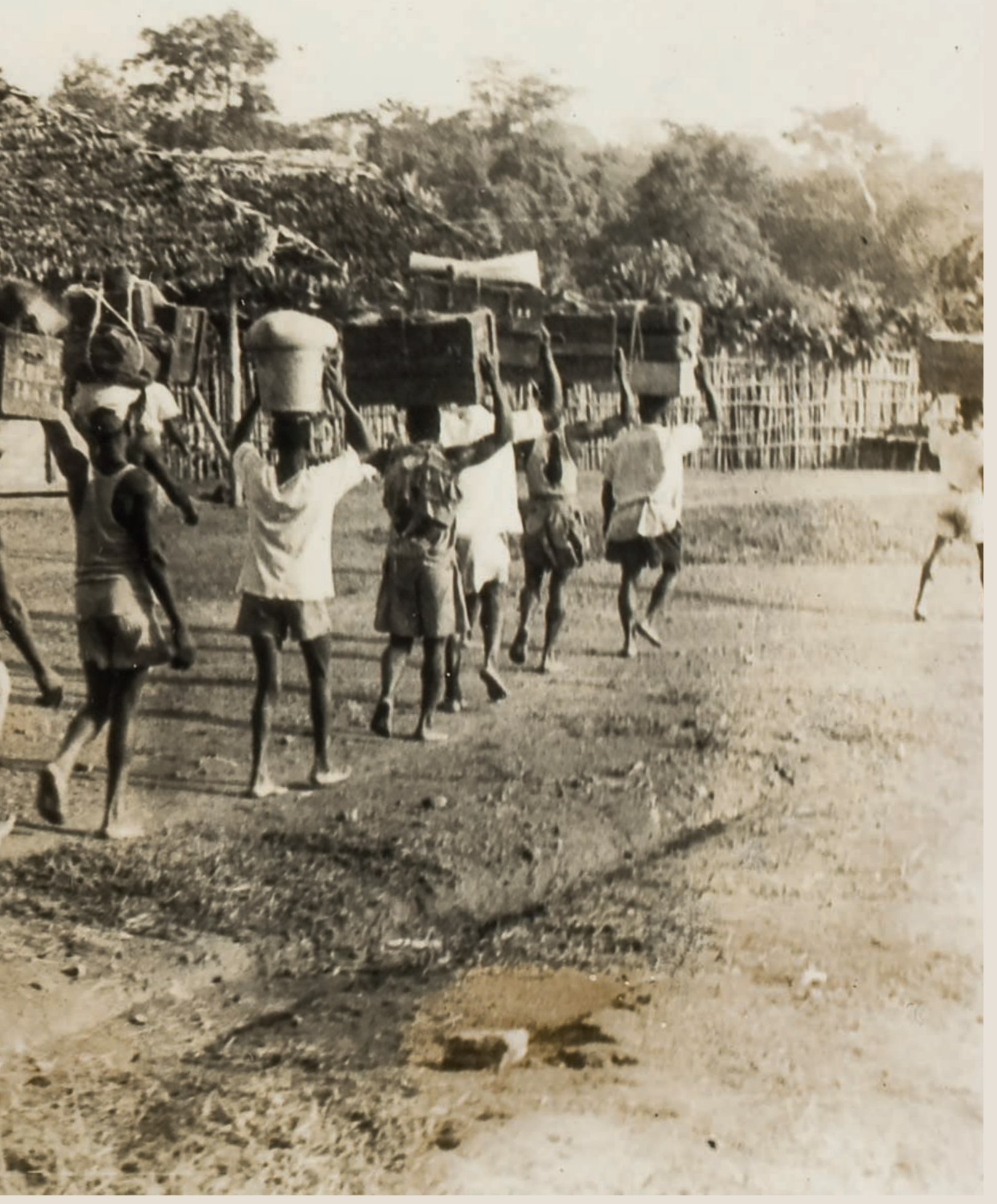


20. Pales et Tassin de Saint-Péreuse 1946 Cette carte a été établie sur la base de 13000 mensurations de la stature, principalement relevées sur des tirailleurs (Léon Pales est médecin lieutenant-colonel et a effectué des mesures sur des tirailleurs marseillais avant la guerre)

21. Pales et Tassin de Saint-Péreuse 1953 Sur l'histoire de cette mission, et plus largement sur la recherche en anthropologie physique dans l'Empire colonial français, voir Sibeud 2012.

\section{double page précédente}

fig. 5

En route. Mission IFAN Dekeyser-Holas au Libéria en 1948. [Porteurs] Photographie Bohumil Holas @ musée du quai Branly. ethnologiques et anthropométriques. Dans sa préface, Monod le présente comme «un recueil de documents, de mélanges ethnologiques» (Monod, in Holas 1952: VIII). Pour Roger Bastide, qui en publie un compte rendu, l'ouvrage présente «l'ensemble [des] fiches» de Holas (Bastide 1952: 166). Ce dernier reconnaît lui-même que cette publication doit "servir de matériaux bruts à des recherches ultérieures plus approfondies" (Holas 1952: IX). Son caractère hybride et hétéroclite trouve une explication dans celui, extensif et itinérant, de la mission. Holas parle ainsi d'«ethnographie lato sensu ».

Les données relatives à la géographie humaine et à l'anthropométrie sont les plus nombreuses: il s'agit pour l'ethnologue d'articuler observation démographique, mesures anthropométriques et comparatisme linguistique. Le projet que Holas formule d'écrire un article intitulé "Observations de la tête chez les peuplades est-libériennes (méthode par sériation)", pour la revue Acta Tropica, témoigne de l'importance qu'il accorde plus spécifiquement à l'anthropométrie. Cet intérêt trouve plusieurs explications.

L'enjeu est d'abord de combler un vide: l'ethnologue constate en effet que, "sur la carte de la stature en AOF établie par Léon Pales ${ }^{20}$, bien que quelques chiffres soient portés en ce qui concerne les territoires étrangers (Guinée portugaise, Sierra Leone, Côte-de-l'Or et Nigéria), le Libéria figure comme une tache blanche " (ibid.: 285). La référence aux travaux de Pales montre bien par ailleurs comment les collectes anthropométriques de Holas et Dekeyser s'inscrivent dans le prolongement de la mission anthropologique de l'AOF dirigée par celui-ci (janvier 1946-août 1948), tentative tardive de «raciologie comparative " des populations colonisées ${ }^{21}$. II y a de nouveau ici l'idée, dans une visée exploratoire, de combler des lacunes et sans doute de répondre à l'appel de Pales, selon qui «l'enquête extensive [en anthropologie physique], en particulier en ce qui concerne la stature, peut être conduite par des observateurs non spécialisés; on ne leur demande que d'être consciencieux» (Pales et Tassin de Saint-Péreuse 1946: 101-102). Dans l'édition des Conseils aux chercheurs de 1948, est d'ailleurs mise en avant l'idée plus générale selon laquelle «l'anthropologie [physique] de l'Afrique est encore à faire " (IFAN 1948).

L'objectif est surtout, pour Holas, de répondre aux demandes de Richard-Molard concernant le chantier des cartes ethnodémographiques dont il a la charge. Dans cette perspective, il s'agit notamment de comparer les «races» de part et d'autre du Cavally, de rechercher les «parentés raciales" ou encore d'apporter des réponses à des questions plus spécifiques, en particulier celle des deux «races kono" - ceci dans le cadre plus général de la comparaison entre les deux types, sénégalais et guinéen (ou «soudanais " et "sylvestre»), définis par Henri-Victor Vallois, alors professeur d'«ethnologie des hommes actuels et fossiles" au Muséum national d'histoire naturelle (Vallois 1941). Comme le note Marie-Albane de Suremain, "l'“indigène de la forêt” était réputé plus attardé et craintif, héritier des traditions de simples chasseurs-cueilleurs, alors que celui de la savane était présenté comme un éleveur ou agriculteur plus précoce" (Suremain 2005: 68). La recherche du «type sylvestre" donne alors en partie sens, pour Holas, au caractère méfiant - voire hostile - des populations rencontrées. Sur le plan scientifique, dans les hypothèses qu'il propose 
au sujet des migrations ethniques, il présente les populations de l'Est libérien comme constitutives d'un isolat, cet isolat servant ensuite de cadre interprétatif pour les données plus spécifiquement ethnologiques ${ }^{\mathbf{2 2}}$.

Dans la pratique, les relevés anthropométriques de l'ethnologue témoignent de leur dimension politique. Les premières mensurations effectuées au cours de la mission sont faites à Tabou, en Côte d'Ivoire, sur «des prisonniers et des prisonnières mis à notre disposition grâce à l'obligeance de l'administration française (M. Passeleau, administrateur en chef des Colonies)" (Holas 1952: 230). Une fois la frontière libérienne franchie, les personnes mesurées sont payées et volontaires (mais Holas insiste de nouveau sur la méfiance des populations locales). Les interférences entre zoologie et anthropologie physique sont également notables: les mêmes instruments de mensuration sont utilisés par Dekeyser pour la mesure des spécimens zoologiques et par Holas pour l'enquête anthropométrique, tandis que les talents d'aquarelliste du premier sont mobilisés à la fois pour figurer les espèces zoologiques et les types humains. Sur le terrain de la mission Dekeyser-Holas, un tel passage du spécimen au type, c'est-àdire de la logique de l'inventaire à celle de la typologie, témoigne là encore d'un cadre naturaliste partagé entre zoologie et anthropologie. II revient ainsi à «zoologiser une partie de l'humanité » (Boëtsch et Chevé 2006: 26).

Au regard des données collectées, celles qui concernent l'ethnologie proprement dite apparaissent tout à fait maigres. Holas s'était notamment fixé pour objectif d'étudier l'ethnologie religieuse, en particulier «le domaine du fétichisme et de l'animisme ${ }^{\mathbf{2 3}}$ ». L'ethnographie qu'il mène pour ainsi dire en passant ne lui permet guère d'avancer sur ce point, comme le note Bastide dans son compte rendu: "La religion par exemple n'est saisie que du dehors, à travers la description de quelques emplacements sacrés ou de quelques cérémonies publiques, vues au hasard du voyage. " (Bastide 1952: 166) Deux cas sont cependant développés sur plusieurs pages. L'un concerne les silures sacrés du village de Flewoke (Holas 1952: 405-407), et l'on retrouve ici l'intérêt déjà indiqué pour le débat concernant le totémisme. Le second met en scène les légendes du lamantin et fournit la matière de l'un des deux articles publiés en 1949 (Holas 1949a), avant l'ouvrage de 1952. Dans ce dernier, ce cas est cependant traité avec les «arts pariétaux» dans la partie consacrée aux «manifestations intellectuelles», et non dans la partie d'ethnologie religieuse (Holas 1952: 429-430). Sur la base de relevés de figurations d'animaux hybrides, Holas propose de témoigner des rapports entre les croyances religieuses et la mise en image. Dans son article, il note qu'il s'agit d'« un lamantin bien imaginaire qui, souvent, dans la fantaisie de son créateur, se transforme en un monstre multiforme et incompréhensible. Ici, en effet, on ne discutera plus la "rigidité de la forme" des arts nègres" (Holas 1949a: 1). Au sujet des mêmes dessins, dans un texte publié plus tard dans un guide de la chasse en AOF, il écrit que "la "licence artistique" semble même surpasser la légende, en s'extériorisant sans bornes» (Holas 1956: 315). On retrouve ici le goût du chasseur pour la faune sauvage, mais on voit aussi comment les enjeux plastiques et cognitifs l'emportent sur l'ethnologie religieuse proprement dite.
22. Holas reproduit en outre la distinction proposée par Maurice Delafosse entre les «indigènes" et les « Noirs à demi européanisés qui vivent au contact des Blancs ou des Libériens, dans les ports et les centres de la côte " (Delafosse 1901: 12, note 2).

23. Lettre de Bohumil Holas à Théodore Monod, 24 septembre 1947, dossier E7.2, archives de l'IFAN-CAD. 


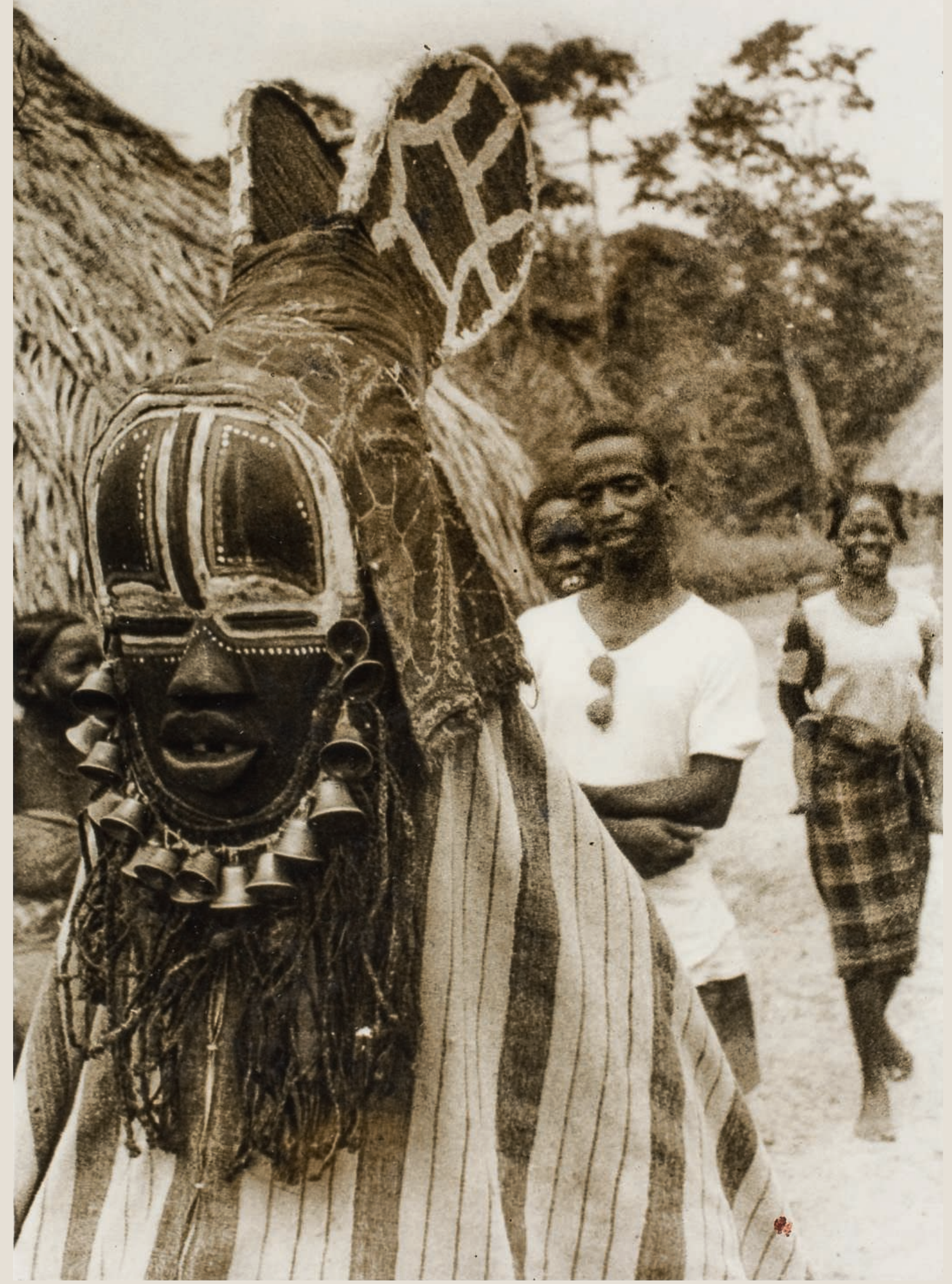


Les pages les plus intéressantes de l'ouvrage de Holas témoignent d'ailleurs de ce goût pour l'art que l'ethnologue ne cessera de développer par la suite, en particulier au musée d'Abidjan dont il est le conservateur de 1947 à 1978. II avertit cependant:

II n'est nullement dans l'intention du présent compte rendu de contribuer à de savantes discussions autour des arts nègres.

Depuis l'époque de Frobenius, cette matière riche et passionnante a été continuellement pétrie par les mains des spécialistes et (hélas!) des amateurs collectionneurs, et largement exploitée, tant bien que mal. (Holas 1952: 415)

Lors de la mission Dekeyser-Holas, la recherche des formes est orientée vers les figurations décoratives (dessins ornementaux sur les murs des habitations) et vers les objets du quotidien. Holas établit un lien étroit entre «corruption » des coutumes et «impureté» du style, en appliquant l'idée d'isolat culturel aux enjeux stylistiques: «Plus on pénètre dans le sein de la forêt, plus on trouve d'œuvres plastiques remarquables, et d'une pureté stylistique supérieure. » (Ibid.: 469) II mentionne à ce propos un «type dégradé » de masque (les notions de «type» ou de "spécimen » sont fréquemment utilisées pour les objets, d'une manière d'ailleurs classique pour ce qui concerne l'ethnographie muséale).

Les collectes ethnographiques de Holas comprennent donc avant tout des objets banals. II respecte en ce sens les «conseils aux chercheurs » de I'IFAN, selon lesquels «une collection ethnographique n'est pas une collection d'œuvres d'art rarissimes. Elle doit, bien au contraire, comprendre avant tout des objets appartenant véritablement à la vie réelle " (IFAN 1948: 58) - écho de la fameuse règle de la boîte de conserve de Marcel Mauss ${ }^{24}$. L'idée est alors de constituer des séries comparatives (ibid.: 59). Pour autant, il y a chez Holas une originalité qui consiste à rechercher dans ces objets ordinaires une dimension esthétique:

Ce sont tout d'abord les masques et les statuettes taillées dans le bois qui attirent, en tant que produits les plus spectaculaires de la statuaire négro-africaine, la curiosité des collectionneurs, et retiennent le plus vif intérêt des auteurs.

Mais, mieux encore que dans ces œuvres frappantes, une expression plus modeste, mais aussi sensible, fine, de la plastique nègre réside dans les menus objets d'utilisation banale. (Holas 1952: 468)

Parmi les modestes ustensiles ménagers, noircis et dégradés par l'usage quotidien, on rencontre parfois des formes d'une grande beauté. (Ibid. : 469)

Holas collecte ainsi des mortiers, des cuillères, des mortiers à tabac, des plats, des peignes, ainsi qu'une épingle à cheveux taillée dans la côte d'un animal que Dekeyser identifie comme étant sans doute «un céphalophe

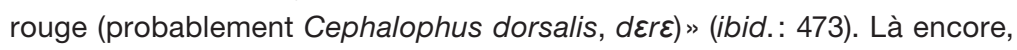
Holas a recours aux services de Dekeyser pour toute une série de déterminations zoologiques. À la date du 19 mars 1948, il écrit ainsi dans son carnet de terrain: «motifs d'animaux (explications zoo-ethnologiques avec
24. Par cette expression, Jean Bazin (2002; voir aussi Jamin 2004) faisait référence à la formule de Michel Leiris, inspiré par Marcel Mauss, selon laquelle "une boîte de conserve, par exemple, caractérise mieux nos sociétés que le bijou le plus somptueux ou que le timbre le plus rare" (Griaule et Leiris 1931: 8).

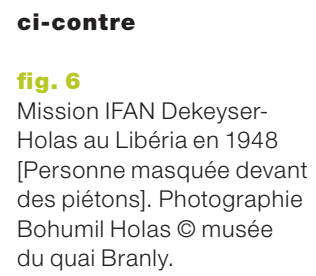


25. Carnet «Mission Libéria 1948 ", le 19 mars 1948, archives du musée du quai Branly.

26. Pour l'ethnologie française, voir en particulier les cas de Marcel Griaule (Jolly 2009) et de Thérèse Rivière (Coquet 2009).

27. Sur ce thème, voir Lefebvre 2011.

\section{ci-contre}

fig. 7

Dessin de sujet libre, exécuté par des élèves krou de Barclayville, in Holas 1952, p. 462.
M. P.L. Dekeyser) dans les arts africains (Libéria ${ }^{25}$ ) '. À propos des dessins relevés sur les murs des maisons et publiés, il indique: "Le zoologiste de notre mission n'a pas hésité pour déterminer l'espèce de ce saurien; c'est un crocodile à museau étroit (Mesocistops cataphractus). » (Ibid. : 423) Les arts pariétaux, considérés comme des "témoignages de la vie intellectuelle du Noir libérien " (ibid.: 437), forment ainsi des supports de production de savoirs multiples, voire des supports de projection.

II n'est pas anodin en effet que Holas accorde une place importante aux dessins d'enfants, à côté des arts pariétaux, dans un double objectif d'interprétation psychologique et d'entreprise comparative, double objectif alors énoncé et appliqué par des ethnologues depuis une vingtaine d'années ${ }^{26}$ (Paget 1932; Anastasi et Foley 1936). Tout au long de la mission, il organise des concours de dessins auprès de groupes d'enfants ou de jeunes adultes scolarisés (généralement dans les missions protestantes), sur deux sujets, l'un imposé et l'autre libre. Le second donne des résultats parfois surprenants puisque sur l'une des planches publiées par Holas figure, parmi plusieurs animaux, un portrait enfantin de Dekeyser, insérant ainsi le zoologiste de la mission dans une sorte de planche zoologique (Holas 1952: 462; fig. 7). II n'est pas étonnant que Bastide ait trouvé dans ces dessins le plus grand intérêt: "Le chapitre sur les dessins d'écoliers pourrait permettre une étude des plus captivantes sur les rapports entre facteurs psychologiques et facteurs sociaux dans les dessins enfantins, en particulier ceux qui omettent d'indiquer le milieu, ou ceux qui représentent des Blancs, ou ceux qui n'oublient pas de dessiner le sexe de personnages pourtant habillés. " (Bastide 1952: 166-167)

Mais plus que les facteurs psychologiques des enfants, ce sont ceux de Holas qu'il est intéressant de questionner. Les sujets imposés sont: «Notre missionnaire prêchant en ville", "Le chef de tribu voyageant dans son hamac", "La musique et la danse dans notre village", "Un Blanc dans notre village" («avec tous les caractères personnels de l'ethnologue de notre mission: cheveux, barbe, ainsi que son accessoire typique, une serviette de cuir", Holas 1952: 439), «Deux hommes blancs dans notre village». Une telle injonction à représenter la situation de rencontre établit une continuité entre plusieurs figures du pouvoir: le missionnaire, le chef de tribu, le zoologiste et l'ethnologue. Mais demander à des enfants ou à de jeunes adultes de figurer la mission Dekeyser-Holas révèle également un certain jeu de projection, narcissique sans aucun doute, puisque l'ethnologue ne cesse de dessiner les indigènes, son collègue zoologiste et lui-même dans les marges de ses carnets de terrain (fig. 8). Il y a là quelque chose du croqueur croqué qui paraît révélateur des pratiques de terrain de Holas et des rapports de pouvoir qu'elles révèlent, mais aussi d'un certain humour de l'ethnologue.

Si la mission Dekeyser-Holas se distingue des autres missions organisées par l'IFAN par le choix d'un terrain non colonial et par l'expérimentation d'une bidisciplinarité originale, sinon novatrice, elle témoigne cependant parfaitement des trois impasses dans lesquelles se trouve l'institut au milieu $\mathrm{du} \mathrm{xx}^{\mathrm{e}}$ siècle. Premièrement, la remise en question du caractère artificiel des frontières se fait uniquement sur une base scientifique ${ }^{27}$. Elle n'interroge à aucun moment la dimension historique et les enjeux politiques de 


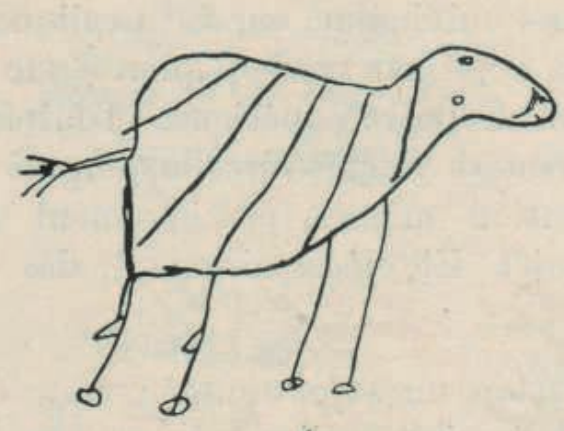

1.

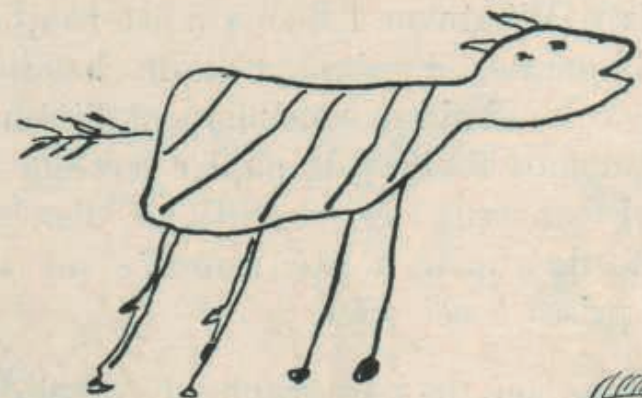

2.

Drawing

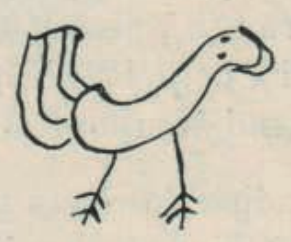

4.
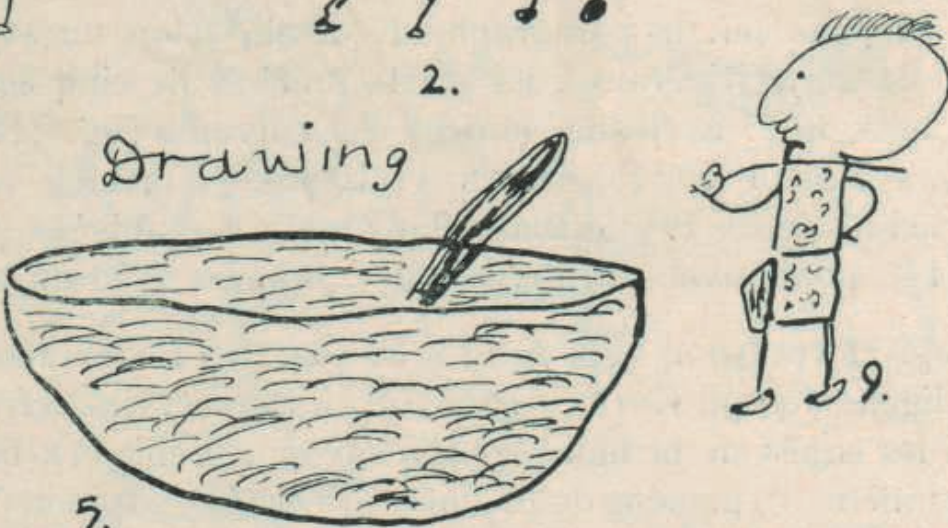

6.
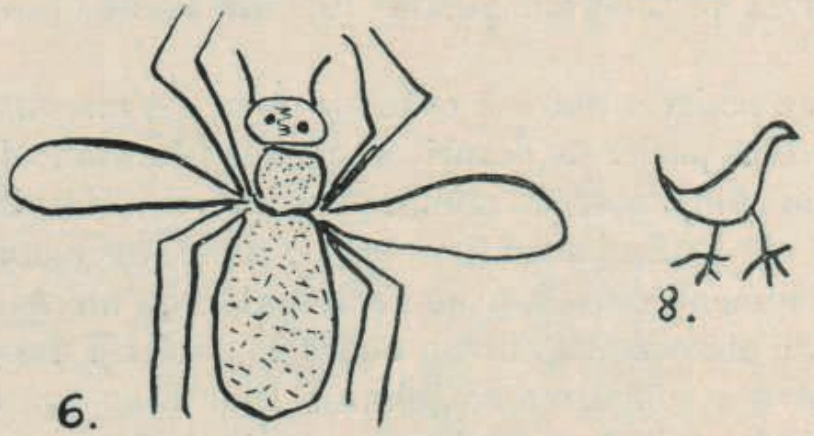

8.
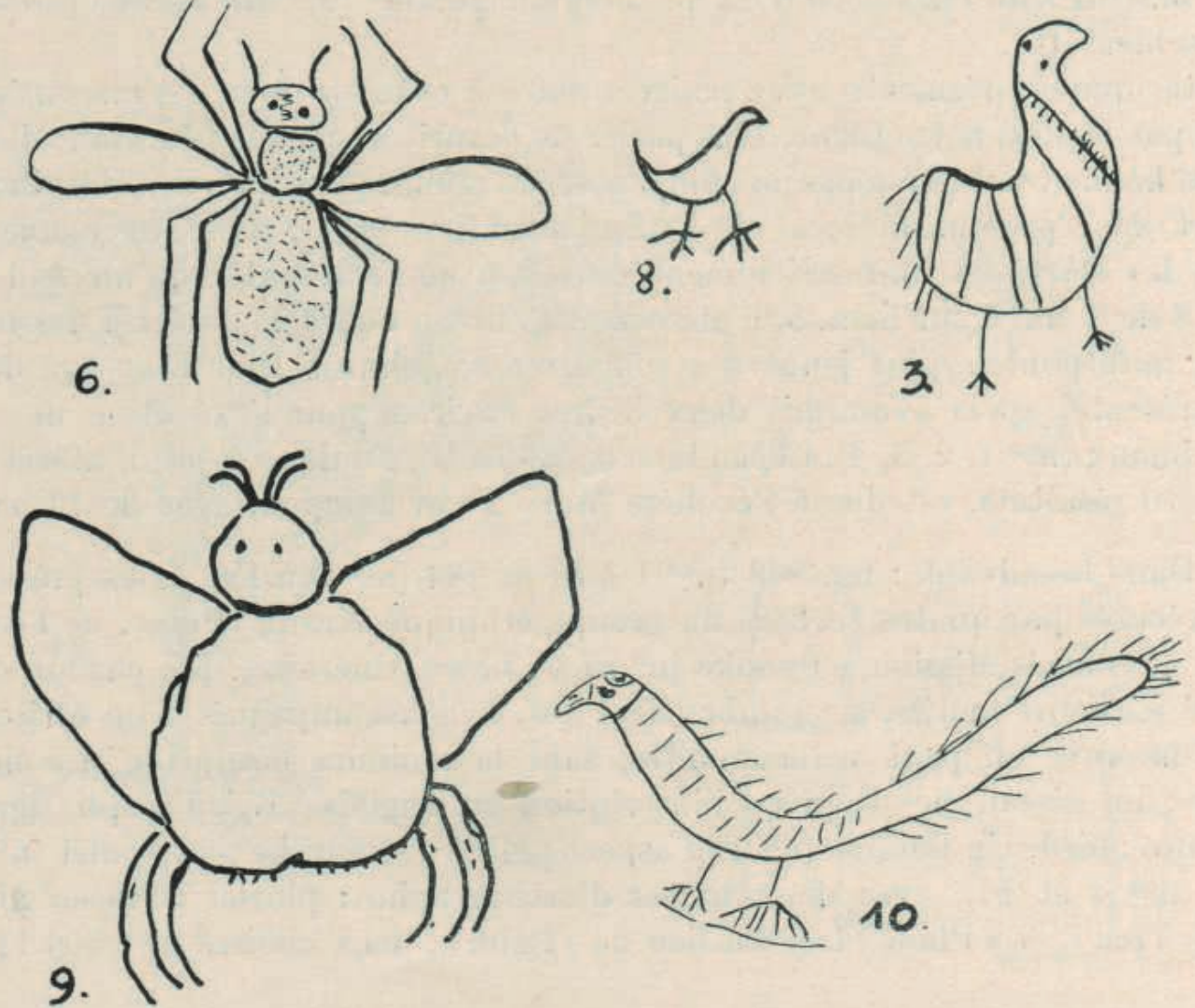

Fig. 242. - Dessin de sujet libre, exécuté par des élèves krou de Barclayville. 
28. De ce point de vue, la mission fournit un exemple parmi de nombreux autres de la redéfinition de l'exploration géographique (le travail de cartographie de la totalité de la planète qui faisait des premiers explorateurs des "découvreurs" touchant à sa fin) en exploration scientifique (voir par exemple Collectif 1952). Cette redéfinition, véritable lieu commun des écrits d'ethnologues entre 1930 et 1960 , doit également être mise en rapport avec la disciplinarisation et la professionnalisation de l'ethnologie. leur tracé, ce qui explique les incompréhensions (et même les énervements) des deux chercheurs concernant les contraintes de la recherche au Libéria, mais aussi et surtout la logique administrative et ethnodémographique qui continue de prévaloir hors du territoire colonial. Deuxièmement, la mise en place d'une mission fortement bidisciplinaire censée traduire la visée encyclopédiste de l'IFAN (et celle du Muséum national d'histoire naturelle en arrière-plan) échoue à produire autre chose que des collections hétéroclites et des connaissances fragmentaires, la démarche extensive pouvant parfois même donner l'impression d'un certain amateurisme. Enfin, et pour ce qui concerne plus spécifiquement l'ethnologie, au-delà du profil atypique de Holas (qui joue d'ailleurs à ce moment son recrutement comme chercheur à I'IFAN), la quête transversale d'une pureté ethnique, religieuse ou stylistique, renforcée par l'idée d'exploration scientifique, témoigne à coup sûr de la fin d'une époque ${ }^{28}$.

Chacune à leur manière, ces trois impasses s'expliquent, sur le plan des pratiques, par les limites de l'application des logiques de production coloniale des savoirs sur un terrain pour ainsi dire indiscipliné, échappant à l'administration coloniale. Dans le Libéria indépendant, l'articulation des formes d'objectivation scientifique et d'objectivation administrative qui caractérise les sciences coloniales (L'Estoile 2000) se retrouve mise à l'épreuve, préfigurant le tournant que provoquent les décolonisations dans l'africanisme français. Ces trois impasses trouvent enfin et surtout une part d'explication dans la prégnance d'un certain paradigme racial, au moment même où l'Unesco initie, en France, un chantier de réflexion critique sur la notion de race qui mobilisera, entre autres chercheurs, des ethnologues aussi fameux qu'Alfred Métraux, Claude Lévi-Strauss et Michel Leiris (Conklin 2013: 327-332). Dans le cadre de la mission Dekeyser-Holas, ce paradigme racial, loin d'être remis en cause, apparaît transversal. II explique à la fois le recours à l'anthropologie physique et à l'ethnodémographie dans la définition du territoire étudié, les convergences entre zoologie et ethnologie et la production conjointe d'un isolat ethnique, d'une pureté religieuse et d'une authenticité artistique. En ce sens, la mission Dekeyser-Holas dans l'Est libérien est bien l'un des lieux où la fabrique des sciences coloniales peut s'observer, dans la mesure où elle vise (sous couvert de mettre en place une coopération avec un État indépendant) à administrer les preuves de l'imbrication du territoire et de la race en la naturalisant, donc en la dépolitisant.

Université Lumière Lyon 2 - Centre de recherches et d'études anthropologiques j.bondaz@univ-lyon2.fr 


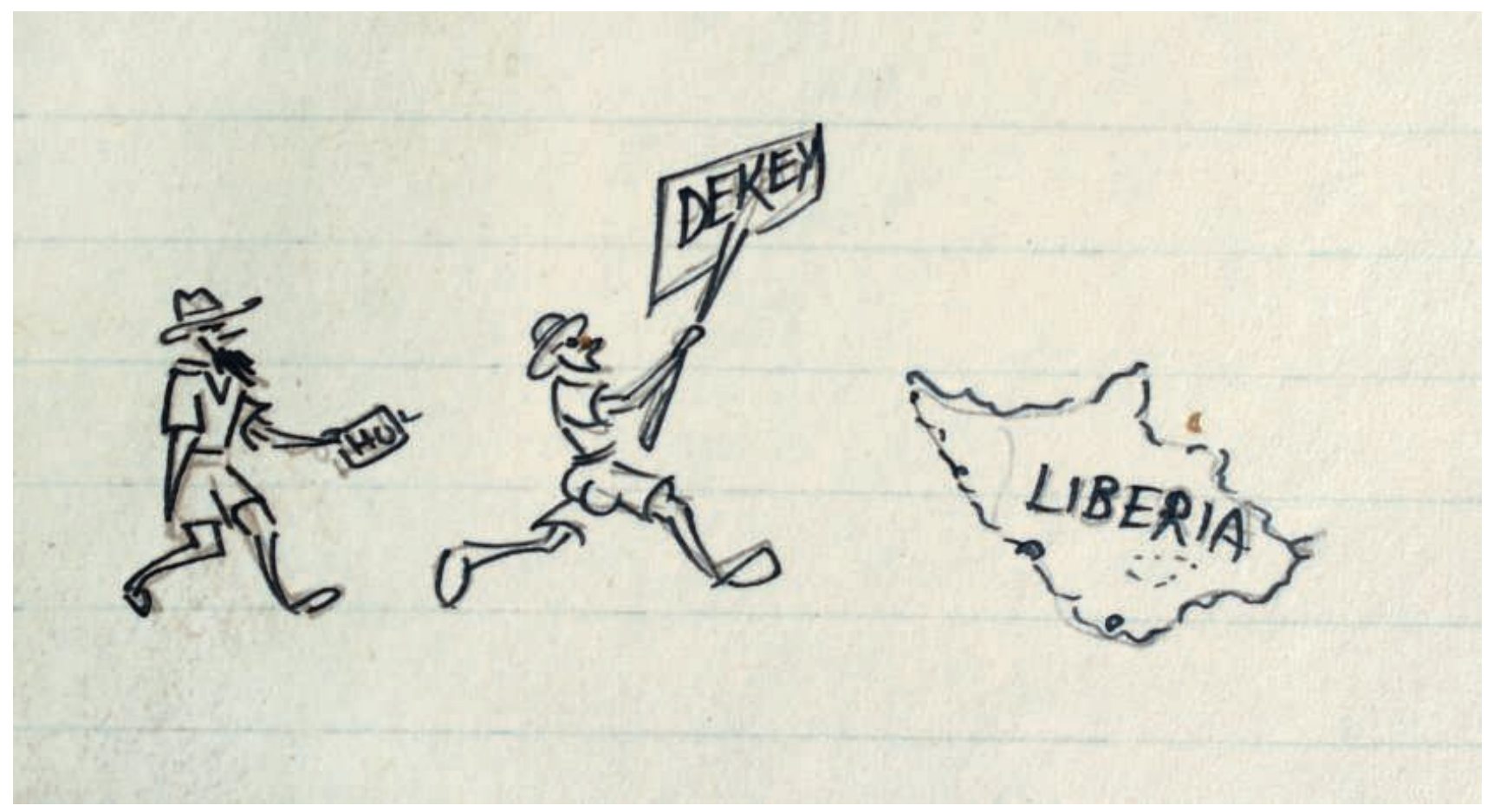

fig. 8

Bohumil Holas, dessin en marge de ses notes du 10 janvier 1948,

"Carnet", fonds

Bohumil Holas,

musée du quai Branly. 


\section{Adedze, Agbenyega}

2003 «In the pursuit of knowledge and power: French scientific research in West Africa ", 1938-65", Comparative Studies of South Asia, Africa and the Middle East 23(1-2): 335-344.

\section{Anastasi, Anne et Foley, John P. Jr}

1936 "An analysis of spontaneous drawings by children in different cultures", Journal of Applied Psychology 20(6) : 689-726.

\section{Balandier, Georges et Mercier, Paul}

1952 Particularisme et évolution. Les pêcheurs lebou du Sénégal. Saint-Louis, IFAN («Études sénégalaises » 3).

\section{Basilewski, Pierre}

1949 «Contribution à l'étude des coléoptères Carabidae de l'Afrique occidentale. 1. Coléoptères carabiques recueillis au Libéria par MM. Dekeyser et Holas ", Bulletin de I'IFAN 11(3-4) : 327-328.

\section{Bastide, Roger}

1952 «Au Libéria

une reconnaissance scientifique ", Annales. Économies, Sociétés, Civilisations 7(4): 565-567.

\section{Bazin, Jean}

2002 "N'importe quoi", in Marc-Olivier Gonseth, Jacques Hainard et Roland Kaehr (éd.) Le Musée cannibale. Neuchâtel, musée d'Ethnographie de Neuchâtel: 273-284.

\section{Boëtsch, Gilles et Chevé, Dominique}

2006 «Du corps en mesure au corps dé-mesuré: une écriture anthropobiologique du corps? ", Corps 1: 23-30.

\section{Bondaz, Julien}

2011 "L'ethnographie comme chasse. Michel Leiris et les animaux de la mission Dakar-Djibouti ”, Gradhiva (n.s.) 13: 162-181.
2013 «L'ethnographie parasitée? Anthropologie et entomologie en Afrique de l'Ouest (1928-1960)", L'Homme 206: 121-150.

2015 "Bêtes de terrain. Savoirs et affects dans l'invention de l'ethnozoologie", Anthropologie et Sociétés 39 (1-2): 37-59.

\section{Büttikofer, Johann}

1890 Reisebilder aus Liberia. Resultate geographischer naturwissenschaftlicher und ethnographischer Untersuchungen während der Jahre 1879-1882 und 1886-1887, 2 vol., Leyde, E.J. Brill.

\section{Chambers, David Wade et Gillespie, Richard}

2000 "Locality in the history of science: Colonial science, technoscience, and indigenous knowledge ", Osiris ( $2^{\mathrm{e}} \mathrm{s}$.) XV: 221-240.

\section{Collectif}

1952 Explorations outre-mer. À travers I'Union française. Paris, La Documentation française.

\section{Conklin, Alice L.}

2013 In the Museum of Man Race, Anthropology, and Empire in France, 1850-1950. Ithaca et Londres, Cornell University Press.

\section{Coquet, Michèle}

2009 "L'" album de dessins indigènes". Thérèse Rivière chez les Ath Abderrahman Kebèche de l'Aurès (Algérie) ", Gradhiva (n.s.) 9: 189-203.

\section{Dekeyser, Pierre Louis}

1954 "À propos des écureuils volants ", Notes africaines 64 : 121-124.

1955 Les Mammifères de l'Afrique noire française. Dakar, IFAN («Initiations africaines» 1).

\section{Dekeyser, Pierre Louis et Villiers, André}

1948 Récolte et préparation des collections zoologiques. Dakar, IFAN.
1955 "Céphalophe à dos jaune et céphalophe de Jentink ", Notes africaines 86 : 54-57.

\section{Delafosse, Maurice}

1901 Les Libériens et les Baoulé. Nègres dits civilisés et nègres dits sauvages. Paris, Librairie africaine et coloniale.

\section{Dieterlen, Germaine}

1952 "Méthode moderne de l'ethnographie ", in Henri Lauga (textes et documents réunis par),

De la banquise à la jungle.

Les Français, la terre et les hommes. Paris, Plon: 165-172.

\section{Dresch, Jean}

1951 "Les publications de l'Institut français d'Afrique noire", Annales. Économies, Sociétés, Civilisations $6(2): 225-227$

\section{Gouvernement général de I'AOF-IFAN}

1950 Première conférence internationale des africanistes de l'Ouest. Comptes rendus, t. I. Dakar, IFAN.

1951 Première conférence internationale des africanistes de l'Ouest. Comptes rendus, t. II. Dakar, IFAN.

\section{Griaule, Marcel et Leiris, Michel}

1931 Instructions sommaires pour les collecteurs d'objets ethnographiques. Paris, musée d'Éthnographie (Muséum national d'histoire naturelle)-mission scientifique Dakar-Djibouti.

\section{Holas, Bohumil}

1949a «Les monstres du Cavally (en marge des légendes du lamantin) ", Notes africaines 41: 1-3.

\section{9b «Deux modèles} de "calendrier de semaine" observés en Libéria (mission P.-L. Dekeyser-B. Holas, 1948), Notes africaines $42: 45$.

1952 Mission dans l'Est libérien (P.L. Dekeyser-B. Holas). Résultats démographiques, ethnologiques et anthropométriques. Dakar, IFAN.
1956 "La faune sauvage, source d'inspiration des arts africains ". in Georges Roure, Faune et chasse en Afrique occidentale française. Dakar, Éditions GIA: 311-321.

1962 Ouvrages et articles 1944-1962. Paris, Librairie orientaliste Paul Geuthner.

\section{IFAN}

1948 Conseils aux chercheurs. Dakar, IFAN.

1954 L'Institut français d'Afrique noire. Dakar, IFAN.

\section{Jacobs, Nancy J.}

2006 "The intimate politics of ornithology in colonial Africa , Comparative Studies in Society and History 48(3) : 564-603.

\section{Jamin, Jean}

2004 "La règle de la boîte de conserve", L'Homme 170: 7-10.

\section{Jézéquel, Jean-Hervé}

2011 «Les professionnels africains de la recherche dans l'État colonial tardif. Le personnel local de l'Institut français d'Afrique noire entre 1938 et 1960 ", Revue d'histoire des sciences humaines 24 : 35-60.

\section{Johnston, Sir Harry}

1906 Liberia. Londres, Hutchinson \& $\mathrm{CO}$

\section{Jolly, Éric}

2009 «Des jeux aux mythes : le parcours ethnographique de Marcel Griaule ", Gradhiva (n.s.) 9. 164-187.

amotte, Maxime, Roy, Roger et Xavier, Françoise

2003 «Les premiers temps de l'étude scientifique et de la protection du Nimba (1942-1978)», in Maxime Lamotte et Roger Roy (éd.), Le Peuplement animal du mont Nimba (Guinée, Côte d'Ivoire, Libéria). Paris, Muséum national d'histoire naturelle: 11-27. 


\section{Lefebvre, Camille}

2011 «La décolonisation d'un lieu commun. L'artificialité des frontières africaines: un legs intellectuel colonial devenu étendard de l'anticolonialisme ", Revue d'histoire des sciences humaines 24: 77-104.

\section{Lepesme, Pierre}

1950 «Longicornes récoltés au Libéria par P.L. Dekeyser et B. Holas ", Bulletin de I'IFAN 12(2) : 395-400.

\section{L'Estoile, Benoît de}

$\mathbf{2 0 0 0}$ "Science de l'homme et "domination rationnelle". Savoir ethnologique et politique indigène en Afrique coloniale française ", Revue de synthèse 3-4 : 291-323.

2005 «Une petite armée de travailleurs auxiliaires: la division du travail et ses enjeux dans l'ethnologie française des années 1930 ", Cahiers du centre de recherche historique $36:$ 31-59.

$\mathbf{2 0 0 7}$ Le Goût des autres. De l'Exposition coloniale aux arts premiers. Paris, Flammarion.

\section{Mahé, Claude}

1958 Au Libéria avec les fils d'esclaves. Namur, Les Éditions du Soleil Levant.

\section{McBride, David}

2002 Missions for Science: U.S. Technology and Medicine in America's African World. New Brunswick et Londres, Rutgers University Press.

\section{Monod, Théodore}

1943 "L'Institut français d'Afrique noire", Africa 14(4) : 194-199.

1947 "La recherche au Libéria", Chronique de l'IFAN 5.

1948a "1948-1958»,

Notes africaines 37.

1948b "A bibliography of Liberia ", Man 48: 69.

1955 «Préface », in Dekeyser 1955 : 11-12.

\section{Paget, G.}

1932 "Some drawings of men and women made by children of certain Non-European races ", Journal of the Royal Anthropological Institute of Great Britain and Ireland 62 : 127-144.

\section{Pales, Léon et Tassin de Saint-Péreuse, Marie}

1946 "La carte de répartition de la stature des populations de l'AOF", Bulletins et Mémoires de la Société d'anthropologie de Paris 7(1-3) : 101-104.

1953 «Raciologie comparative des populations de l'AOF : stature, indice cormique et indice céphalique en Afrique occidentale ", Bulletins et Mémoires de la Société d'anthropologie de Paris 4(3-4) 185-497.

\section{Picard, Jacques}

1950 «Hesperiidae du Libéria collectés par P.L. Dekeyser et B. Holas ", Bulletin de I'IFAN 12(3): 625-628.

\section{Schiebinger, Londa}

2005 «Forum introduction: The European colonial science complex", Isis $96:$ 52-55.

\section{Schnell, Raymond}

1948 "À propos de l'hypothèse d'un peuplement négrille ancien de l'Afrique occidentale", L'Anthropologie 52: 229-242.

\section{Seth, Suman}

2009 «Putting knowledge in its place: Science, colonialism, and the postcolonial ", Postcolonial Studies 12(4) : 373-388

\section{Sibeud, Emmanuelle}

2012 "A useless colonial science? Practicing anthropology in the French colonial empire, circa 1880-1960", Current Anthropology 53(5) numéro spécial: S83-S94.

\section{Stempffer, Henry}

1950 « Contribution à l'étude des Lycaenidae du Liberia ", Bulletin de I'IFAN 12(2) : 402-407.

\section{Strong, Richard P. (éd.)}

1930 The African Republic of Liberia and the Belgian Congo Based on the Observations Made and the Material Collected during the Harvard African Expedition, 1926-1927, 2 vol. Cambridge, Harvard University Press.

\section{Suremain, Marie-Albane} de

2004 «Faire du terrain en AOF dans les années cinquante", Ethnologie française 34(4) : 651-659.

2005 «Inventaire ou savoirfaire? À la recherche d'identités "ethnogéographiques" en Afrique de l'Ouest au lendemain de la Seconde Guerre mondiale », in Chantal Chanson-Jabeur et Odile Goerg, Mama Africa. Hommage à Catherine Coquery-Vidrovitch. Paris, L'Harmattan : 57-73.

2008 "Cartes

ethnodémographiques de l'Afrique de l'Ouest: enjeux d'une construction", in Mappemondes, "Géographie et colonisation ", coord. par Hélène Blais et Florence Deprest, 92(4) numéro spécial [En ligne]; http://mappemonde.mgm.fr/num20/ articles/art08404.html.

\section{Vallois, Henri-Victor}

1941 «Recherches

anthropologiques sur les Peuls et divers Noirs de l'Afrique occidentale d'après les mensurations de M. Leca (mission Labouret, 1932)", Bulletins et Mémoires de la Société d'anthropologie de Paris 9(2): 20-74.

\section{Villiers, André}

1949 Mission P.L. Dekeyser et A. Villiers en Guinée et Côte d'Ivoire (1946). Insectes (première partie). Dakar, IFAN

1950 "Hémiptères récoltés au Libéria par P.L. Dekeyser et B. Holas ", Bulletin de I'IFAN 12(4) 930-939.

page 168 et ci-contre Mission IFAN Dekeyser-Holas au Libéria en 1948 [Personne masquée devant des piétons]. Photographie Bohumil Holas (c) musée du quai Branly. 